\title{
AUTOMATING USABILITY EVALUATION OF VISUAL USER INTERFACES IN THE TILE LOGIC
}

\author{
Vladimir V. Devyatkov and Evgeny A. Tipsin \\ Bauman Moscow State Technical University, Russia
}

\begin{abstract}
This article presents the methodology of formal automated evaluation of user interface usability. The tiles logic was selected as the language for solving problems in formal usability verification. The paper contains an overview of the related works, explains the reasons for using the tile logic, and considers the principles of verification (proof) of usability as validation of agent's interaction. The differences and advantages of the developed approach from the well-known are shown. A description of the agents interaction in the tile logic is used to automate usability evaluation. The methodology of formal automated usability evaluation is demonstrated by describing the interaction of two simple agents in the tile logic. In conclusion, we present future of use and development of the proposed approach.
\end{abstract}

\section{KEYWORDS}

Tiles Logic, Verification of Properties, Graphical User Interface, Usability, Usability Evaluation, Intelligent Agents, Automating Usability Evaluation, Human-Machine Interactions

\section{INTRODUCTION}

There was introduced the term of usability in the standards ISO / IEC 25010, which is defined as "convenience and ease of use, degree of convenience", as well as suitability of use, ergonomics (the ability of an application to be understood, be studied, be used and attractive for a user in the specified conditions to achieve a set goals with necessary efficiency, effectiveness and satisfaction.

There is a wide range of traditional means of usability evaluation: standards/guidelines review, heuristic evaluation, cognitive walkthrough, pluralistic walkthrough, feature inspection, task analysis (Hackos and Redish, 1998), focus groups (Nielsen, 1997), surveys, interviews, questionnaires and benchmarking, eye tracking (Jacob and Karn, 2003), analysis of user's electroencephalogram (Alfimtsev et al., 2015) and others. However, all these usability evaluations are rather labor-intensive. This is reflected in spending time for preparing for benchmarking, selection of respondents and further analysis of obtained results by the experts of human-machine interaction. In some cases, availability of special equipment is necessary. In order to reduce complexity and amount of manual work, the direction of automated usability evaluation is relevant. In this direction, there are three basic approaches to automating evaluation of visual user interfaces usability:

1. Analysis of user logs properties (Approach 1). Analysis of logs (files) that contains entries of user's actions in process of interaction with real applications or dynamic prototypes in chronological order. Typically, these actions are motion of a mouse cursor; a time spent on page, transition between pages and other.

2. Parametric analysis of page properties (Approach 2). The analysis of parameters the particular pages of the application for compliance with the best practices in design, visual noise and interface complexity, also identifying the page areas that the user will draw attention primarily (Alfimtsev et al., 2016).

3. Model-oriented proof of the properties existence. (Approach 3) In the paper (Devyatkov, 2016) considered the properties of multimodal intelligent interfaces, implementation of which, according to the authors, provides a "barrier-free" user interaction with application (Sakulin et al., 2018).

In the first column of table lis the list, according to an analytical review, containing 10 selection criteria an approach to usability evaluation. In the other columns, for each approach, a "+"sign indicates that criterion 
is met in approach, and a " - " sign indicates that criterion is not met. This table shows that the most number of criteria is met in the model-oriented approach. There are three criteria that is not met: the explanation of user's behavior, no need to invite human-machine interaction experts, the lack of the subjective factor.

Despite the dissatisfaction of the three criteria, the model-oriented approach is preferable. Model-oriented approach uses models of architecture and system behavior in which interaction is implemented. If the model developer believes, that its architecture and behavior has all necessary objective properties, that provide necessary degree of usability, then remains to prove that this architecture and behavior is met of these properties.

The authors believe that the address these drawbacks (explanation of user behavior, no need to invite human-machine interaction experts, the lack of a subjective factor) is possible by automated model-oriented proof of objective property presences in architecture and interacting models of user behavior and an application within adequate calculus, including:

- formal language of adequate patterns description of user behavior and executor in interaction process;

- formal description of the objective properties of the architecture and interacting models behavior that ensures a high degree of usability in this language;

- many consistent inference rules in this language;

- output strategy to ensure completeness proof of architecture properties and interacting models behavior.

A formal description of objective architecture properties and interacting models behavior, that ensures high degree of usability, will obviate need to invite human-machine interaction experts and, as a result, their subjective opinion (Chernenkiy et al, 2018).

Table 1. Approaches and criteria

\begin{tabular}{|l|c|c|c|}
\hline \multicolumn{1}{|c|}{ Approaches } & 1 & 2 & 3 \\
\hline Criteria & & & \\
\hline Explanation of user behavior & - & - & + \\
\hline Ease of use rating after making changes to the interface & - & + & - \\
\hline No need to invite human-machine interaction experts & - & - & - \\
\hline Time executions & - & + & + \\
\hline Considering the context of using the application & - & - & + \\
\hline User experience considering & - & - & + \\
\hline Dynamic page analysis & + & - & + \\
\hline The lack of the subjective factor & - & - & - \\
\hline
\end{tabular}

A strategy for ensure completeness proof of architecture properties and interactive models behavior based on inference rules will allow to identify an application usability and disadvantage reasons of usage of application.

Automation of output strategy will ensure ease of evaluating usability after changes in interaction process.

According to the author's opinion, language of tile logic is the most appropriate to automation of evaluation usability. In particular, this opinion is based, on the fact that a tile, as a construction, allow to display the elements of pages naturally and transitions between the elements, implemented as a result of certain user actions. In addition, acceptable tiles sequences of user interaction with system can be put together, according to certain rules, that serve as a means of usability evaluation.

The principles of proving any properties of systems based on the tiles logic have been known for a long time (Bruni et al, 1998). In order to create a language of the tile logic for our case, it is necessary to create a basic tiles set that allow to represent application behavior (Ivashkin et al, 2018). Further in this article, an application will be called the agent-executor and a respondent will be called the agent-user.

Below are the basic concepts of the tile logic. Then a formal description of application (agent-executor) and respondent (agent-user) models will be provided. After that, usability criteria will be introduced. The example of usability evaluation of the application will be shown. In conclusion, directions of further work of automation usability evaluation of applications are given. 


\section{THE TILES AND TILE OPERATIONS}

Rules structure of inference is an advantage of the tile logic, each of rules can be represented as a tile. A tile structure in general form is shown on Figure 1.

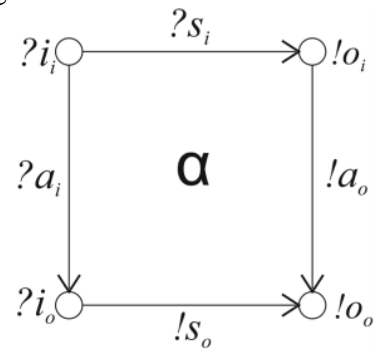

Figure 1. The tile: $\alpha$ - the tile name; ? $s_{i}$ - the tile initial configuration; $! s_{o}$ - the tile final configuration; $? a_{i}-$ the tile perception; $! a_{o}-$ the tile reaction; $? i_{i}-$ the tile initial input interface; $? o_{i}-$ the tile initial output interface;

$? i_{o}-$ the tile final input interface; $! o_{o}-$ the tile final output interface.

Tile allows to describe system components behavior that contain variables called configurations. This behavior is described in terms of possible interactions with the external or internal environment. System behavior is a coordinated interaction of individual tiles. Tile interfaces define interaction format with the external environment. Tiles can be composited horizontally (denoted by symbol *), vertically (denoted by symbol $\bullet$ ), and in parallel (denoted by symbol $\otimes$ ) to define complex behavior of systems. The tile logic is obtained by introducing a set of basic tiles and a certain set of auxiliary tiles. Tile operations can be performed leading to various kinds of tile compositions, in particular, in order to prove the user interfaces properties.

\section{AGENT ARCHITECTURE FOR AUTOMATION USABILITY EVALUATION}

An architecture, consisting of the agent-executor and the agent-user, is used to demonstrate method of automated usability evaluation process (Vorotnikov et al., 2018).

The agent-executor $(A E)$ has two channels. The input channel $(U E)$ is the output to the agent-user and the output channel is the input to the agent-user $(E U)$. The agent-user can put a message to the input channel of the agent-executor. The agent-executor can take this message. The agent-executor can put a message to the agent-user on it output channel. Further, the agent-executor and agent-user will be called the executor and the user.

If the channels $U E$ and $E U$ compare to the variable $c$ (from channel), and the messages in channels compare to the variable $m$ (from message), then the set of all channel states is $c(m)$, where $c \in\{U E, E U\}$, $m \in\{m(U E) \cup m(E U)\}, m(U E)$ - the set of messages in the channel $U E, m(E U)$ - the set of messages in the channel $E U$. The couple $\left[U E\left(m_{u}\right), E U\left(m_{e}\right)\right]$ is a configuration. If no matter message in the channel $E U$ for the executor, then configuration will be denoted as $\left[U E\left(m_{u}\right), E U\left(\_\right)\right]$, and conversely, if no matter message in the channel $U E$ for the user, then configuration will be denoted as $\left[U E\left(\_\right), E U\left(m_{e}\right)\right]$.

The executor performs internal behavior in accordance with own convictions after receiving a message from the user. The executor and the user interaction begins after receiving a notification of start of interaction from the user. If the executor has concurred to interaction, then it continues. In other case, an interaction terminate. The user stops an interaction by sending an appropriate message to the executor.

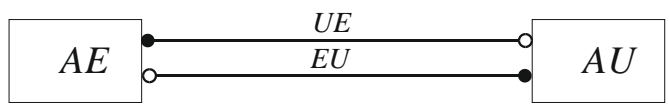

Figure 2. User and executor interaction channels 


\section{USABILITY EVALUATION CRITERIA}

Usability evaluation criteria can be qualitative and quantitative and allow to conclude about interface usability, as well as comparing user interface with other projects or previous versions of the design.

According to Nielsen's approach (Nielsen, 1994), there are three types of evaluation:

- completed the task without difficulty - 100\%;

- had difficulties, but completed the task - 50\%;

- did not complete the task - $0 \%$.

The problem that we placed in this article does not include a detailed consideration of all evaluation criteria. Therefore, in article will be focus on calculus of tile logics.

\section{MODEL OF THE USER INTERFACE}

An application can be represented as a collection of pages. Each page has own user interface, which includes various types of elements: navigation elements, specifying possible movements between pages; control elements to perform actions, such as sending an email, attaching files, or changing content; content items.

Thus, each user interface consists of certain interface elements - primitives of a graphical user interface that have a standard appearance and perform standard actions. Interface elements include: edit fields (textbox), buttons, radio buttons, checkboxes, icons, and others.

Related elements grouping is using to improve usability, for example: alignment, grouping, highlighting, indents, pins, element sizes and spacing. Usually grouped interface elements are named as blocks. Introduce the following notation to proceed to the developed approach specification with the tile logic.

An application $I$ consists of a finite set of pages $P$. A page $p \in P$ consists of at least one block $b \in B$, which may consist of blocks $b_{1}^{\prime}, \ldots, b_{j}^{\prime} \subset b$ and/or interface elements $e_{1}, \ldots, e_{k} \in E e_{1}, \ldots, e_{k} \in b$. Thus, application can be represented as $I=\{P, B, E\}: P$ - a set of pages, which contains the application $\left\{p_{1}, \ldots, p_{k}\right\} \in P ; B-$ a set of blocks $\left\{b_{1}, \ldots, b_{m}\right\} \subset B ; E-$ a set of interface elements $\left\{e_{1}, \ldots, e_{t}\right\} \in E$.

Each page $p \in P$ has the following attributes: $c_{1}^{p}-$ a name of the page; $c_{2}^{p}-$ a set of blocks $\left\{b_{1}, \ldots, b_{n}\right\} \in B$ that contains page.

Each block $b \in B$ has the following attributes: $c_{1}^{b}-$ a set of blocks $\left\{b_{1}^{\prime}, \ldots, b_{j}^{\prime}\right\} \subset B$, that contains the block $\left\{b_{1}, \ldots, b_{j}\right\} \subset b ; c_{2}^{b}-$ a set of interface elements $\left\{e_{1}, e_{2}, \ldots, e_{k}\right\} \subset E$, that contains the block $\left\{e_{1}, e_{2}, \ldots, e_{k}\right\} \subset b$.

Each interface elements $e \in E$ has the following attributes: $c_{1}^{e}-$ type that can be one of eight values: text, input (single line input field), textarea (multiline input field), button, image, select (drop-down list), video, audio; $c_{2}^{e}$ - hint/ user interface label; $c_{3}^{e}$ - content; $c_{4}^{e}$-size, represented by the corresponding pair of values, in the format (width, height); $c_{5}^{e}-$ form; $c_{6}^{e}-$ color; $c_{7}^{e}-$ brightness; $c_{8}^{e}-$ an additional attribute that can contain a description of reactions when interacting with an element, for example, notification of incorrect format of entered data in text field (Sakulin et al., 2018).

\section{AGENT-EXECUTOR REPRESENTATION IN THE TILE LOGIC}

The executor is on interface object, receiving messages from the user, it changes contents of objects (inserts data) or moves to another object.

In order to set the executor behavior, horizontal and vertical tiles compositions are applied. A horizontal composition simulate an action on side of the executor. A vertical composition is to exchange messages with the user (receiving and placing messages in interaction channels).

The executor behavior depends on perception (message from the user in his input channel). It performs a reaction (place message to the user in output channel) in response to communication. The executor analyzes 
the current configuration $\left[U E\left(m_{u}\right), E U\left(\_\right)\right]$(the "Getting an action on an object" tile), perform perception of message $m_{u}$ from the channel $U E\left(m_{u}\right)$, sets the initial output interface $s_{-} i\left(m_{u}\right)$ (from set interface) depending on message $m_{u}$, as a result, the configuration $\left[U E\left(m_{u}\right), E U\left(\_\right)\right]$remains unchanged.

The «Object reaction» (Figure $3 b$ ) tile describes the reaction on message in the output channel. The tile perception is not set. It perform reaction (place message in output channel to the executor) in response. The executor analyzes the current configuration $\left[U E\left(m_{u}\right), E U\left(\_\right)\right]$, generates a reaction $m_{e}$ (message) that puts into the channel $E U\left(m_{e}\right)$, as a result, the configuration $\left[U E\left(m_{e}\right), E U\left(\_\right)\right]$goes into the configuration $\left[U E\left(m_{u}\right), E U\left(m_{e}\right)\right]$.

Blocks and interface elements have a similar description, so let's call them general concept - object.

The user and application interaction may be the following in real situation. The user scans each new page and clicks on the first link similar to that he were looking for. If a result did not meet expectations, user goes back to the previous page and continues scanning (Mahyavanshi et al, 2017). Thus, main types of user interaction with application can be identified: a transition from one object to another; performing actions on an element (click, double click, entering data); return to previous page.
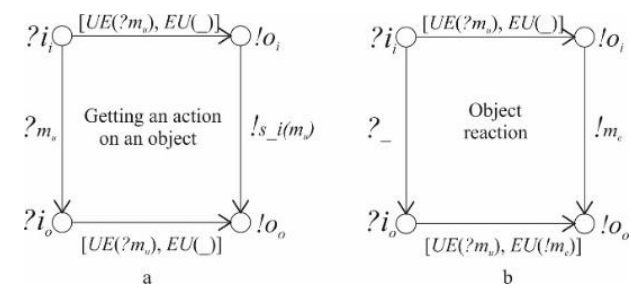

Figure 3. Agent-executor tiles

left - move to left object of the current object; up - move to upper object of the current object; right - move to right object of the current object; down - move to bottom object of the current object; sin gle_click - single click on the object; double_click - double click on the object, back - return to previous page, data - data to enter into interface element.

Perceptions single_click, double_click are available only for interface elements $E$.

A message (reaction) can be the following type: object_inf $o$ - a set of interface object characteristics (block, interface element); notification - notification of correct or erroneous entry of data into interface element.

\section{THE SELECTION PRINCIPLES OF THE AGENT- USER}

Define user groups, to which an application is focused, is performed in selection an user for usability testing. Agent user is a collective character of users group. Agent-user must have behavioral features of selected user group. Agent tiles should take into account features, as well as the goals that need to be identified and reflected by selected user. Thus, the user is a model of behavior for a certain users group, and behavior characteristics are represented in the tile logic. Tile or set of tiles describes characteristics.

In addition to the tiles of behavior patterns, the user have a certain set of attributes that represent the necessary information to interaction. For example, the user should to know a login and a password, which are agent's attributes, when it authorizing in an application. Without these attributes, the user cannot complete a task. Given applications and tasks diversity, it is impossible to set all possible user attributes in advance. Therefore, attributes is set expertly. 


\section{AGENT-USER IN THE TILE LOGIC}

In this paper, the simple user behavior, which receives a message from the executor (the «Receiving object» tile), will demonstrate. The user goes to another interface element (puts the message on the output channel with the transition direction) or puts the data on the interface element (puts the message on the output channel with the data), depending on the message. Transition to another element (the «Performing action» tile) is performed if the message is a notification of correct data entry into an element or interface object description that is not an input field. Otherwise, it is entered data into interface element (the «Goal achievement» tile). Tiles, describing various characteristics and user behavior patterns, will not be considered in this article.

Horizontal and vertical composition is applied to set user behavior, similar the executor. Horizontal composition is an action on side of the user. A Vertical composition is exchange of messages with the executor (receiving and putting messages in interaction channels).

The following five tiles are used to describe user behavior.

1. As stated earlier, the executor and the user interactions begins after receiving notification of start of interaction from the user. The «Interaction initialization» (figure 4a) describes the placement of a message of start of interaction from the user to the executor. The user analyzes the current configuration [EU(_), $\left.U E\left(\_\right)\right]$, generates a response $m_{u}$ (notification of the start of interaction), which is placed in the $U E$ channel, as a result, the configuration $\left[E U\left(\_\right), U E\left({ }_{-}\right)\right]$goes into the configuration $\left[E U\left(\_\right), U E\left(m_{u}\right)\right]$.

2. The «Receiving object» (figure $4 b$ ) tile describes the receipt of a message from the executor. Similarly, the user uses a perception (message) in the input channel placed by the executor. Perception sets the initial output interface and determines the subsequent agent behavior. The initial output interface imposes restrictions on horizontal composition with other tiles that limits the user's acceptable actions. The user analyzes the current configuration $\left[E U\left(m_{e}\right), U E\left(\_\right)\right]$, perform the perception of the message $m_{e}$ from the channel $E U\left(m_{e}\right)$, establishes interfaces $s_{-} i\left(m_{e}\right)$ (from set interface) depending on message, as a result, the configuration $\left[E U\left(m_{e}\right), U E\left(\_\right)\right]$remains unchanged.

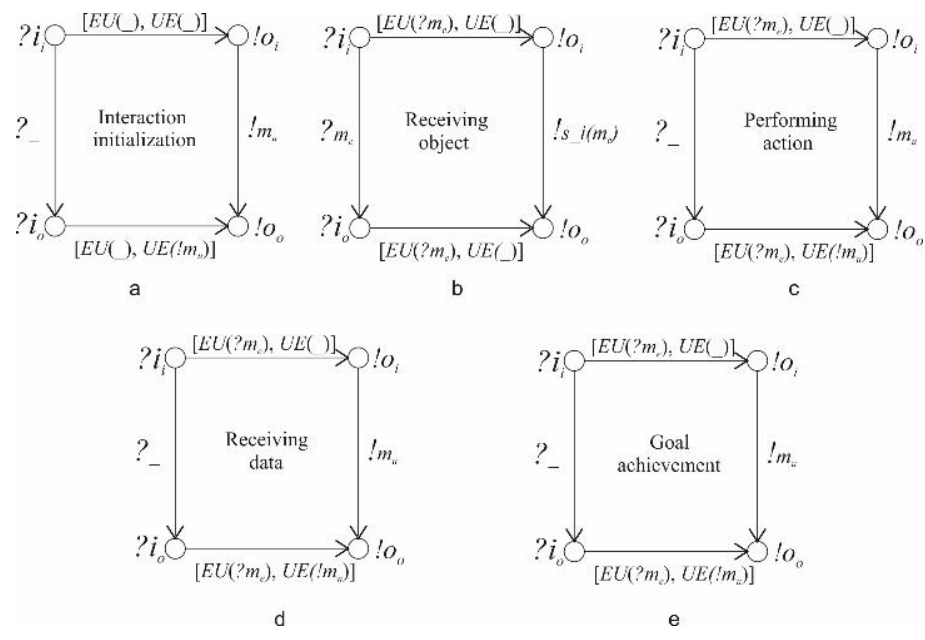

Figure 4. Agent-user tiles

3. The «Performing action» (figure 4c) tile describes the placement in the output channel of transition direction to another interface element. The tile perception is not set. The reaction is to put a message in output channel to the executor. The user analyzes the current configuration $\left[E U\left(m_{e}\right), U E\left(\_\right)\right]$, generates a reaction $m_{u}$ (message) that puts into the channel $U E\left(m_{u}\right)$, as a result, the configuration $\left[E U\left(m_{e}\right), U E\left(\_\right)\right]$ goes into the configuration $\left[E U\left(m_{e}\right), U E\left(m_{u}\right)\right]$. 
4. The «Receiving data» (figure 4d) tile describes the data acquisition for entering into an interface element. The tile perception is not set. The reaction is to put a message in output channel to the executor. The user analyzes the current configuration $\left[E U\left(m_{e}\right), U E\left(\_\right)\right]$, generates a reaction $m_{u}$ (message) that puts into the channel $U E\left(m_{u}\right)$, as a result, the configuration $\left[E U\left(m_{e}\right), U E\left(\_\right)\right]$goes into the configuration $\left[E U\left(m_{e}\right), U E\left(m_{u}\right)\right]$.

5. The «Goal achievement» (figure 4e) tile describes check the goal achievement. The tile perception is not set. The reaction is to put a message in output channel to the executor. The user analyzes the current configuration, generates a reaction $m_{u}$ (message) that puts into the channel $U E\left(m_{u}\right)$, as a result, the configuration $\left[E U\left(m_{e}\right), U E\left(\_\right)\right]$goes into the configuration $\left[E U\left(m_{e}\right), U E\left(m_{u}\right)\right]$.

\section{SIMPLE EXAMPLE OF AUTOMATING USABILITY EVALUATION IN TILE LOGIC}

In this section, the simple example of usability evaluation, that demonstrate a general approach to automating usability evaluation, are shown.

The developed approach of automation of usability evaluation has been demonstrated for the one-page application (figure 5). The application is a service for translating numbers between systems of positional numbers. Application page contains 5 interface elements: page name $e_{1}$; input field for the initial number system $e_{2}$; input field for source number $e_{3}$; input field for the number system to which the initial number will be translated $e_{4}$; output field for result of conversion $e_{5}$. After filling in the fields $e_{2}, e_{3}, e_{4}$ the initial number is transferred to the specified numeral system, the result is placed in the field $e_{5}$.

A developer, who performs usability evaluation, presents an application as a set of tiles. More detailed an application, the higher accuracy of usability evaluation, because each element can affect user behavior. The application can be represented as $I=\{P, B, E\}$, where $P=\left\{p_{1}\right\} ; B=\left\{b_{1}\right\}, b_{1} \subset p_{1} ; E=\left\{e_{1}, \ldots, e_{5}\right\}$, $\left\{e_{1}, \ldots, e_{5}\right\} \subset b_{1}$.

$p_{1}$

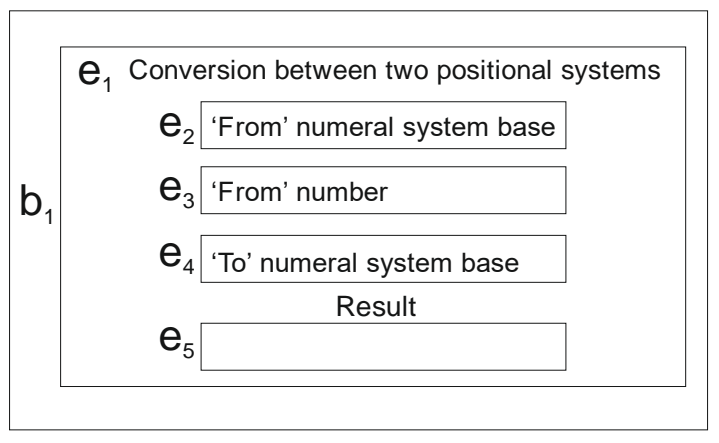

Figure 5. User interface

The sequence of actions is given below, that an expert should perform:

1. The creation of a set of tiles and attributes for each page $p_{1}$, block $b_{1}$ and interface element $e_{1}, \ldots, e_{5}$.

2. Choice of usability criteria to evaluate application. In this paper, only one criterion is considered, which usability evaluation based on result of task (completed - an application has a high degree of usability, otherwise low).

3. Assigning a task to the user to solve it by interacting with the executor.

4. A user type determines user behavioral characteristics. Various types of user agents (behavior model of user's specific groups) are not considered in this article. The user interacts with the executor through the tiles presented in the previous section. 
5. Setting necessary attributes of the user to solve task. For this application: "From" numeral system base -5 ; "From" number - 10; "To" numeral system base -2 .

Consider a sequence of composition of the executor and the user tiles as they interact.

After the user has put a message to the channel $U E$, the user is described by the «Getting an action on an object» tile. Similarly, the executor has put a message to the channel $E U$, the executor is described by the «Receiving object» tile.
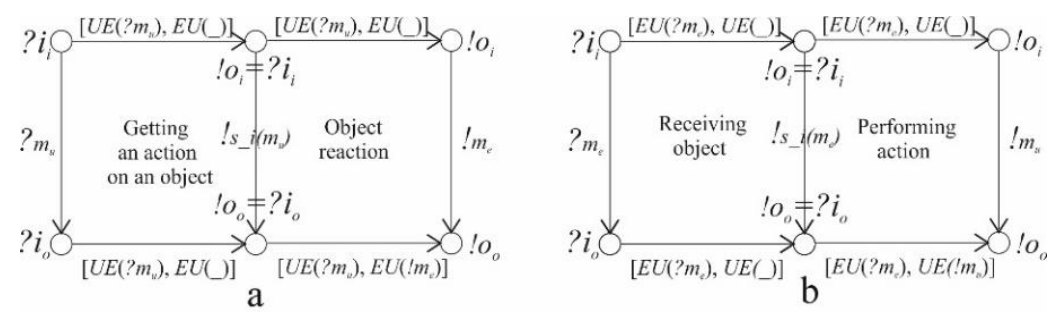

Figure 6. Tiles composition

User (step 1). «Initialization interaction»

Executor (step 2). «Getting an action on an object»* «Object reaction», as it shown on the figure 6a $\left(e_{1}\right)$.

User (step 3). «Receiving object» * «Performing action», as it shown on the figure $6 \mathrm{~b}$.

Executor (step 4). «Getting an action on an object»* «Object reaction», as it shown on the figure 6 a $\left(e_{2}\right)$.

User (step 5). «Receiving object» * «Receiving data»

Executor (step 6). "Getting an action on an object» * «Object reaction», as it shown on the figure 6a.

User (step 7). «Receiving object» * «Performing action», as it shown on the figure $6 \mathrm{~b}$.

Further steps 4-7 are performed similarly for the elements $e_{3}, e_{4}$.

Executor (step 8). «Getting an action on an object» * «Object reaction», as it shown on the figure 6a.

User (step 9). «Receiving object» * «Goal achievement»

According to the usability criteria, it can be concluded that the interface has a high degree of usability.

\section{CONCLUSIONS}

In this paper provides an analytical review of approaches to automating usability evaluation of applications, in which the advantages and disadvantages of each approach were identified. The formal model-oriented method of automated usability evaluation based on tile logic was proposed to address existing deficiencies, in which an application and user model is created. Valid user interaction sequences with an application are assembled from tiles according to certain rules that provide a means for usability evaluation. The use of the method was shown by the example of the one-page application. In future work, the application model will be improved, namely, the addition of new tiles describing transitions between interface elements. Full-on user model, describing characteristics and behavior while scanning the application page, will be created in the tiles logic. User model behavior will compare with the behavior of a real user. Number of usability evaluation metrics will be increased. After the improvements, the usability of the multi-page application will be evaluated. In fact, automating usability in the tile logic is the knowledge extraction of the interacting agents. User behavior is the subject of analytics in a wide variety of applications. It is demand at the present stage of intelligent information systems development. This paper outlines the principles of interacting agents behavior based on the use of processes local behavior patterns in the tile logic. Composition of tiles forms the interacting agents general behavior. The presentation of principles is carried out at a general level, allowing the tiles composition, but without formal proof of properties of the interacting agents actual behavior. In [Devyatkov, 2016], a properties proof concretization is given for the case of proving the presence of intelligent interfaces certain properties and the proof implementation in the logic language PROLOG. Since the basis of the proof procedures in the PROLOG language is unification. Unification can serve as a means of 
detecting the presence of specific behavior in the tiles composition. In addition, by analogy with the same work, if it is know that interacting agents behavior are most interested, then the properties of this behavior can be formulated in the modal logics languages, and then they will be checked. In the future, it is supposed to be implemented.

\section{REFERENCES}

Alfimtsev, A. N., Basarab, M. A., Devyatkov, V. V., \& Levanov, A. A. (2015). A new methodology of usability testing on the base of the analysis of user's electroencephalogram. Journal of Computer Sciences and Applications, 3(5), 105-111.

Alfimtsev, A., Sakulin, S., \& Levanov, A. (2016). Formalization of expert knowledge about the usability of web pages based on user criteria aggregation. International Journal of Software Innovation (IJSI), 4(3), 38-50.

Bruni, R., Meseguer, J., \& Montanari, U. (1998). Process and term tile logic.

Chernenkiy, V., Gapanyuk, Y., Terekhov, V., Revunkov, G., \& Kaganov, Y. (2018). The hybrid intelligent information system approach as the basis for cognitive architecture. Procedia computer science, 145, 143-152.

Devyatkov V.V. Verification of Intelligent Interface Properties in the Tiles Logic. Vestn. Mosk. Gos. Tekh. Univ. im. N.E. Baumana, Priborostr. [Herald of the Bauman Moscow State Tech. Univ., Instrum. Eng.], 2016, no. 3, pp. $65-87$.

Hackos, J. T., \& Redish, J. (1998). User and task analysis for interface design.

Ivashkin, Y. A., Blagoveschensky, I. G., \& Nikitina, M. A. (2018). Neural network and agent technologies in the structural-parametric modeling of technological systems. In 2018 School-Seminar on Optimization Problems and their Applications, OPTA-SCL 2018 (pp. 169-180).

Jacob, R. J., \& Karn, K. S. (2003). Eye tracking in human-computer interaction and usability research: Ready to deliver the promises. In The mind's eye (pp. 573-605).

Mahyavanshi, N., Patil, M., \& Kulkarni, V. (2017). Enhancing Web Usability using User Behavior and Cognitive Study. International Journal of Computer Applications, 164(2).

Nielsen, J. (1997). The use and misuse of focus groups. IEEE software, 14(1), 94-95.

Sakulin, S., Alfimtsev, A., Solovyev, D., \& Sokolov, D. (2018). Web page interface optimization based on nature-inspired algorithms. International Journal of Swarm Intelligence Research (IJSIR), 9(2), 28-46.

Sakulin S. A., Alfimcev A. N., Tipsin E. A. (2018). Verification of user interface distribution based on $\pi$-calculus. Bulletin of Bryansk state technical university, (9), 56-65.

Vorotnikov, S., Ermishin, K., Nazarova, A., \& Yuschenko, A. (2018). Multi-agent Robotic Systems in Collaborative Robotics. In International Conference on Interactive Collaborative Robotics (pp. 270-279). Springer, Cham. 